\title{
A NOTE ON THE MATRIX RENEWAL FUNCTION
}

\author{
A. M. KSHIRSAGAR ${ }^{1}$ and Y. P. GUPTA \\ (Received 10 February 1970; revised 27 July 1970) \\ Communicatcd by B. Mond
}

\begin{abstract}
The Laplace-Stieltjes Transform $m(s)$ of the matrix renewal function $M(t)$ of a Markov Renewal process is expanded in powers of the argument $s$, in this paper, by using a generalized inverse of the matrix $I-P_{0}$, where $P_{0}$ is the transition probability matrix of the imbedded Markov chain. This helps in obtaining the values of moments of any order of the number of renewals and also of the moments of the first passage times, for large values of $t$, the time. All the results of renewal theory are hidden under the Laplacian curtain and this expansion helps to lift this curtain at least for large values of $t$ and is thus useful in predicting the number of renewals.
\end{abstract}

\section{Introduction}

Let $P_{0}=\left[p_{i j}\right](i, j=1,2, \cdots, m)$ be the transition probability matrix of the imbedded Markov Chain of a Markov Renewal Process (M.R.P.), involving a finite number $m$ of states. Let $F_{i j}$ be the distribution function (d.f.) of the time spent in state $i$ by the process before going to the next transitionstate $j(i, j=1,2, \cdots m)$ Let $J_{t}$ denote the state of the process at time $t$ and $N_{j}(t)$ denote the number of visits to state $j$ in the interval $(0, t)$. The matrix $M(t)=\left[M_{i j}(t)\right]$, where

$$
M_{i j}(t)=E\left\{N_{j}(t) \mid J_{0}=i\right\}
$$

is called the matrix renewal function of the M.R.P. Its Laplace-Stieltjes transform (L.-S.T.) is given by Pyke [6], [7] as

$$
m(s)=\int_{0}^{\infty} e^{-s t} d_{t} M(t)=(I-q(s))^{-1}-I
$$

where

$$
Q(t)=\left[Q_{i j}(t)\right], Q_{i j}(t)=p_{i j} F_{i j}(t), q(s)=\int_{0}^{\infty} e^{-s t} d_{t} Q(t) .
$$

The asymptotic behaviour of the renewal function in an ordinary renewal process $(m=1)$ is well-known (see for example Cox [1] or Smith [9] and the simplest way of deriving it is to expand its Laplace transform in powers of the argument $s$,

1 This research was sponsored by the office of Naval Research, Contract No. N00014-68-A0515 project No. NR 042-260. 
in the neighborhood of $s=0$ and use Tauberian arguments. Since all higher order moments of the number of renewals in an ordinary renewal process are expressible in terms of the renewal function, their asymptotic behaviour can also be investigated from this, provided a sufficient number of terms in the expansion of the LaplaceStieltjes transform are retained.

These results and methods can be extended to an M.R.P. The present authors demonstrated this by expanding the L.-S.T. $(I-q(s))^{-1}-I$ given by $(1.2)$ in powers of $s$. The expansion was obtained from

$$
I-q(s)=I-P_{0}+s P_{1}-\frac{s^{2}}{2 !} P_{2}+\frac{s^{3}}{3 !} P_{3}-\cdots
$$

where

$$
P_{k}=\int_{0}^{\infty} x^{k} d Q(x),(k=0,1,2, \cdots)
$$

subject, of course, to the existence of these moments. Hereafter we assume that all these moments exist. The main difficulty in this is obviously the singularity of the matrix $I-P_{0}$. The required expansion of $m(s)$ was obtained in terms of certain matrices $H_{r}(r=0,1,2, \cdots)$ obtainable from the adjoint of $I-P_{0}+s P_{1}$. Recently Hunter [2] and Keilson have independently solved this problem by different methods. Hunter uses a generalized inverse (Rao [8]) of $I-P_{0}$ along with the relationship between the moments of $Q_{i j}$ and $G_{i j}$, the d.f. of the first passage time of the M.R.P. from state $i$ to state $j$ (Pyke [6], [7]). Keilson uses the spectral decomposition of the matrix $q(s)$ and then finds the limiting values of the first two derivatives of $(I-q(s))^{-1}$ with respect to $s$, at $s=0$, which are nothing but the coefficients of powers of $s$ in its expansion. In the present paper, the generalized inverse method is used directly on $(I-q(s))^{-1}$. Investigation of higher moments of $N_{j}(t)$ requires more than two terms in the expansion of $(I-q(s))^{-1}$ and these can be obtained more easily and directly by the method of this paper.

\section{Generalized inverse of $I-P_{0}$}

Pyke [6], [7] has described the classification of the states of a M.R.P. in much the same manner as is done for Markov Chains. Following his terminology, we assume that all the states of the M.R.P. are positive and recurrent. The imbedded Markov Chain is thus regular.

Let $\bar{U}^{\prime}=\left[U_{1}, U_{2}, \cdots, U_{m}\right]$ be the vector of the stationary state probabilities $\left(\sum U_{i}=1\right)$ of the imbedded Markov Chain and let $\bar{e}^{\prime}=[1, \cdots, 1]$, a vector of $m$ components. Then it is well-known that

$$
P_{0} \bar{e}=\bar{e}, \quad \bar{U}^{\prime} P_{0}=\bar{U}^{\prime} .
$$

Let

$$
L=\bar{e} \bar{U}^{\prime},
$$


and

$$
Z=\left(I-P_{0}+L\right)^{-1} \text {. }
$$

$Z$ is known as the fundamental matrix of the Markov Chain (Kemmeny and Snell [4]) and the following results are easily derivable (Hunter [2]).

(i) $Z$ is a generalized inverse of $I-P_{0}$ i.e.

$$
\left(I-P_{0}\right) Z\left(I-P_{0}\right)=\left(I-P_{0}\right)
$$

(ii) $P_{0} Z=Z P_{0}$

$$
\begin{aligned}
& \text { (iii) } Z \bar{e}=\bar{e} \\
& \text { (iv) } \bar{U}^{\prime} Z=\bar{U}^{\prime} \\
& \text { (v) } Z L=L Z=L \\
& \text { (vi) } Z\left(I-P_{0}\right)=I-L
\end{aligned}
$$

We also define

$$
k_{r}=\bar{U}^{\prime} P_{r} \bar{e} \quad(r=1,2, \cdots),
$$

so that

$$
L P_{r} L=k_{r} L \quad(r=1,2, \cdots)
$$

We shall also need the following result (Rao 1966).

The general solution of the equation $A X=B$ is

$$
X=A^{-} B+\left(I-A^{-} A\right) W
$$

where $A^{-}$is any generalized inverse of $A$ and $W$ is any arbitrary matrix. This result holds if and only if the consistency condition

$$
A A^{-} B=B
$$

is satisfied.

\section{Expansion of $(I-q(s))^{-1}$}

It is well-known from the theory of ordinary renewal processes (see for example Keilson [3]) that, if the Markov chain is ergodic and if the $P_{k}$ 's exist, $(I-q(s))^{-1}$ can be expanded in the form

$$
\frac{1}{s} A_{-1}+A_{0}+s A_{1}+s^{2} A^{2}+\cdots
$$

We shall now show how the $A$ 's can be determined. From (1.4),

$$
\left(I-P_{0}+s P_{1}-\frac{s^{2}}{2 !} P_{2}+\cdots\right)\left(\frac{1}{s} A_{-1}+A_{0}+s A_{1}+\cdots\right) \equiv I .
$$


Equating coefficients of $s^{r}$ on both sides,

$$
\left(I-P_{0}\right) A_{r}-\sum_{\alpha=1}^{r+1} \frac{(-1)^{\alpha}}{\alpha !} P_{\alpha} A_{r-\alpha}=\delta_{r 0} I,(r=-1,0,1,2, \cdots)
$$

where $\delta_{r 0}$ is the kronecker delta. Premultiply (3.3) by $L$ and observing that $L\left(I-P_{0}\right)=0$, we obtain

$$
L D_{r+1}=0, \quad(r=-1,0,1, \cdots)
$$

where

$$
D_{r+1}=\sum_{\alpha=1}^{r+1} \frac{(-1)^{\alpha}}{\alpha !} P_{\alpha} A_{r-\alpha}+\delta_{r 0} I
$$

Write (3.3) as an equation in $A_{r}$, in the form

$$
\left(I-P_{0}\right) A_{r}=D_{r+1} \quad(r=-1,0,1, \cdots) .
$$

We shall now verify that the consistency condition (2.8) is satisfied for this equation. For this we need (i) and (vi) of (2.4) viz that $Z$ is a generalized inverse of $I-P_{0}$ and that $Z\left(I-P_{0}\right)=(I-L)$. The consistency condition is then

or which is the same as,

$$
Z\left(I-P_{0}\right) D_{r+1}=D_{r+1}
$$

$$
(I-L) D_{r+1}=D_{r+1},
$$

which is true, on account of (3.4). The general solution of (3.6) is, therefore, by $(2.7)$

$$
A_{r}=Z D_{r+1}+L W_{r}, \quad(r=-1,0,1, \cdots)
$$

where $W_{r}$ is some arbitrary matrix. We shall now obtain the value of $W_{r}$. From (3.4), $L D_{r+2}=0(r=-2,-1,0,1, \cdots)$, i.e.

$$
L\left\{\sum_{\alpha=1}^{r+2} \frac{(-1)^{\alpha}}{\alpha !} P_{\alpha} A_{r+1-\alpha}+\delta_{r+1} I_{0}\right\}=0
$$

i.e.

$$
-L P_{1} A_{r}+\sum_{\alpha=2}^{r+2} \frac{(-1)^{\alpha}}{\alpha !} L P_{\alpha} A_{r+1-\alpha}+\delta_{r+1,0} L=0 .
$$

Substitute for $A_{r}$ in this equation from (3.7). We get

$$
-L P_{1}\left(Z D_{r+1}+L W_{r}\right)+\sum_{\alpha=2}^{r+2} \frac{(-1)^{\alpha}}{\alpha !} L P_{\alpha} A_{r+1-\alpha}+\delta_{r+1,0} L=0 .
$$

From (2.2) and (2.5), we obtain

$$
\begin{aligned}
L P_{1} L & =e U^{\prime} P_{1} e U^{\prime} \\
& =\left(U^{\prime} P_{1} e\right) e U^{\prime} \\
& =k_{1} L,
\end{aligned}
$$


and so, (3.8) yields

$$
L W_{r}=-\frac{1}{k_{1}} L P_{1} Z D_{r+1}+\frac{1}{k_{1}} \sum_{\alpha=1}^{r+1} \frac{(-1)^{\alpha+1}}{(\alpha+1) !} L P_{\alpha+1} A_{r-\alpha}+\frac{1}{k_{1}} \delta_{r+1,0} L .
$$

Substituting this back in (3.7), we get finally the general solution of (3.6) as

$$
\begin{aligned}
A_{r}= & \sum_{\alpha=1}^{r+1} \frac{(-1)^{\alpha}}{\alpha !}\left\{Z P_{\alpha}-\frac{1}{k_{1}} L P_{1} Z P_{\alpha}-\frac{1}{k_{1}(\alpha+1)} L P_{\alpha+1}\right\} A_{r-\alpha} \\
& +\delta_{r 0}\left(I-\frac{1}{k_{1}} L P_{1}\right) Z+\frac{1}{k_{1}} \delta_{r+1,0} L,(r=-1,0,1, \cdots) .
\end{aligned}
$$

Since this general solution does not include any arbitrary matrix, it is the only unique solution and in particular,

$$
\begin{aligned}
A_{-1}= & \frac{1}{k_{1}} L . \\
A_{0}= & \left(I-\frac{1}{k_{1}} L P_{1}\right) Z\left(I-\frac{1}{k_{1}} P_{1} L\right)+\frac{k_{2}}{2 k_{1}^{2}} L, \\
A_{1}= & \left\{-Z P_{1}+\frac{1}{k_{1}} L P_{1} Z P_{1}+\frac{1}{2 k_{1}} L P_{2}\right\} A_{0} \\
& +\frac{1}{2 k_{1}}\left\{Z P_{2}-\frac{1}{k_{1}} L P_{1} Z P_{2}-\frac{1}{3 k_{1}} L P_{3}\right\} L .
\end{aligned}
$$

\section{Remarks}

This expansion of $(I-q(s))^{-1}$ yields immediately, the following asymptotic expression for the renewal function $M(t)$, by inverting the L.-S.T. for large $t$ :

$$
M(t)=t A_{-1}+A_{0}+0(1) .
$$

The Laplace-Stieltjes transform of the second factorial moment $E\left\{N_{j}(t) \overline{N_{j}(t)-1}\right\}$ is given by (Kshirsagar and Gupta [5])

$$
2\left\{( I - q ( s ) ^ { - 1 } - I \} _ { d } \left\{\left(I-q(s)^{-1}-I\right\}\right.\right.
$$

where ${ }_{d} B$ stands for the diagonal matrix obtained from a matrix $B$, by removing all the off-diagonal elements. By using (3.7), (3.1), in (4.2), it can be readily shown that, for large $t$, the second factorial moment, conditional on $J_{0}=i$ is of the form

$$
t^{2} G_{1}+t G_{2}+G_{3}+0(1) \text {. }
$$

The matrices $G_{1}, G_{2}, G_{3}$ can be easily obtained in terms of $A_{-1}, A_{0}, A_{1}, \cdots$ and then in terms of $P_{k}$ 's and $Z, L$ from (3.7). Extension to higher order moments is also possible in exactly a similarly manner, as their L.-S.T.'s all turn up in terms of $(I-q(s))^{-1}$ and $q(s)$. 


\section{References}

[1] D. R. Cox, Renewal Theory (Methuen, London, 1962).

[2] Jeffrey J. Hunter, On the moments of Markov renewal processes (University of North Carolina, Institute of Statistics Mimeo Series No. 589, 1968).

[3] J. Keilson, 'On the matrix renewal function for Markov renewal processes', Ann. Math. Statist. 40 (1969), 1901-1907.

[4] J. G. Kemeny and J. L. Snell, Finite Markov Chains (Van Nostrand, New York, 1960).

[5] A. M. Kshirsagar and Y. P. Gupta, 'Asymptotic values of the first two moments in Markov Renewal Processes' Biometrika 54 (1967), 597-603.

[6] Ronald Pyke, 'Markov Renewal processes: Definitions and preliminary properties' Ann. Math. Stat. 32 (1961), 1231-1241.

[7] Ronald Pyke, 'Markov Renewal processes with finitely many states', Ann. Math. Statist. 32 (1961), $1243-1259$.

[8] C. Radhakrishna Rao, (1966) Generalized inverse for matrices and its applications in mathematical statistics (Research papers in Statistics, Festschrift for J. Neyman, Wiley, New York, 1966).

[9] W. L. Smith, 'On the cumulants of renewal processes' Biometrika, 46 (1959), 1-29.

Southern Methodist University

Dallas, Texas, U.S.A.

and

Delhi University

India 